\section{Five-year incidence and progression of diabetic retinopathy in a defined older population: the Blue Mountains Eye Study}

L Cikamatana, P Mitchell, E Rochtchina, S Foran and JJ Wang

\section{Abstract}

Aims To determine 5-year incidence and progression of diabetic retinopathy in an older Australian population-based cohort. Methods During the period 1992-1994, the Blue Mountains Eye Study examined 3654 residents aged $49+$ years $(82.4 \%$ of those eligible), living in two urban postcode areas, west of Sydney, Australia. Participants were subsequently invited to attend 5-year follow-up exams. After excluding 543 (14.8\%) who died during the follow-up period, 2334 persons $\mathbf{( 7 5 . 0 \% )}$ were re-examined during 1997-1999. The examination included a comprehensive questionnaire, blood pressure measurement, standardised refraction, Zeiss stereo retinal photographs, and estimation of fasting blood glucose. Diabetic retinopathy was graded from the retinal photographs, using the modified Early Treatment Diabetic Retinopathy Scale classification (15-step scale). Results Of participants with diabetes diagnosed at baseline, 150 were re-examined, including 139 with gradable fundus photographs. The cumulative 5-year incidence of diabetic retinopathy was $22.2 \%$ before $95 \%$ confidence interval (CI) $14.1-32.2 \%$.

Retinopathy progression (1+ steps) was documented in $25.9 \%$ (95\% CI $18.8-34.0 \%$ ) of participants with retinopathy and gradable photographs at both visits; in $58.3 \%$ of these cases, a $2+$-step progression was documented. Progression to proliferative retinopathy occurred in only $4.1 \%$ of those with retinopathy at baseline. The only baseline risk factors associated with retinopathy progression, after adjusting for age and gender, were increase in fasting blood glucose, odds ratio (OR) 1.2 (95\% CI 1.1-1.4)/ $\mathrm{mmol} / \mathrm{l}$, and increase in diabetes duration, OR 2.3 (95\% CI 1.0-5.3)/10 years.

Conclusions These data provide 5-year cumulative incidence of diabetic retinopathy in a defined older population. Increase in diabetes duration and elevated baseline fasting blood glucose level predicted retinopathy incidence. Eye (2007) 21, 465-471. doi:10.1038/sj.eye.6702771; published online 23 February 2007

Keywords: type II diabetes; diabetic retinopathy; incidence; population-based study; Blue Mountains Eye Study

\section{Introduction}

With the increasing prevalence of type II (non-insulin dependent) diabetes in most Western countries associated with the ageing population, the ocular morbidity from diabetes is likely to remain an ongoing public health issue in the future.

The Wisconsin Epidemiologic Study of Diabetic Retinopathy (WESDR) reported a 4-year cumulative retinopathy incidence of $32.7 \%$ for diabetic subjects not using insulin in the age group 60-74 years, whereas for persons using insulin, the incidence was $45.9 \% .^{1}$ Few community-based studies of older populations have reported diabetic retinopathy incidence. In the Beaver Dam Eye Study, Wisconsin reported on the prevalence of diabetic retinopathy, ${ }^{2}$ but to date, have not reported diabetic retinopathy incidence. In clinic-based studies, ${ }^{1,3-8}$ a wide variation in diabetic retinopathy incidence has
Department of Ophthalmology, University of Sydney, Sydney, Australia

Correspondence: P Mitchell, Department of

Ophthalmology (Centre for Vision Research, Westmead Millennium Instuitute) Westmead Hospital, Hawkesbury Rd, Westmead, New South Wales 2145, Australia

Tel: + 6129845 7960; Fax: + 61298458345 E-mail: paul_mitchell@ wmi.usyd.edu.au

Received: 2 August 2005 Accepted: 17 August 2005 Published online: 23 February 2007 
been reported, presumably because different criteria have been used to diagnose diabetes, to define and grade retinopathy, and because there have been both variable follow-up periods and sample selection.

We aimed to determine 5-year incidence of diabetic retinopathy among persons with diabetes (both previously detected and newly diagnosed) from a defined older community and the 5-year progression and regression of existing diabetic retinopathy lesions that were detected at the baseline examination.

\section{Methods}

\section{Population studied}

The Blue Mountains Eye study (BMES) is a populationbased study of vision and common eye diseases in a defined older community of two postcode areas in the Blue Mountains region, west of Sydney, Australia. Baseline examinations were conducted between 1992 and 1994 (BMES-1) and 5-year follow-up examinations between 1997 and 1999 (BMES-2). The study methodology has been described in detail previously., ${ }^{9,10}$ Briefly, after a door-to-door census in the study region, permanent non-institutionalised residents born before 1 January 1943 were invited to the local hospital to undergo a detailed eye examination. Of the 4433 eligible residents, 3654 participated $(82.4 \%), 501$ refused (11.35\%), whereas $210(4.85 \%)$ had moved from the study area, and $68(1.5 \%)$ had died after the census was carried out. Residents participating in the initial study were invited after 5 years for a follow-up examination at the same local hospital. At follow-up, 2334 of the 3654 baseline participants (63.9\%) were re-examined, $543(14.8 \%)$ had died, $394(10.8 \%)$ refused to participate, and $383(10.5 \%)$ had moved from the study area. ${ }^{11,12}$

\section{Procedures}

Similar procedures were conducted at both the baseline and 5-year follow-up visits, including

- Detailed interview by trained interviewers covering both ocular and systemic medical history and medication usage.

- Comprehensive eye examination with measures of height, weight, and seated blood pressure.

- $30^{\circ}$ fundus photographs taken using a Zeiss FF3 fundus camera (Carl Zeiss, Oberkochen, Germany) through dilated pupils, including stereo-photographs of Diabetic Retinopathy Study (DRS) fields 1 (centred on the optic disc) and 2 (centred on the macula) and nonstereo photographs of fields 3 (temporal), 4 and 5 (upper and lower vascular arcades, respectively), and a field nasal to the optic disc. Fields 4 and 5 were modified to accommodate the vascular arcade slightly closer to the centre of each photograph. ${ }^{10}$

- Fasting pathology tests for blood glucose, serum lipids, creatinine, and fibrinogen levels, within 8 weeks of the clinic visit. A fasting blood glucose level $\geqslant 7.0 \mathrm{mmol} / 1$ (hexokinase method) was used to diagnose new cases of diabetes mellitus. ${ }^{13}$

Examination findings and recommendations were sent to each participant, and his/her general practitioner and eye care practitioner.

\section{Grading}

Fundus photographs of all participants with diabetes diagnosed by history or fasting blood glucose $\geqslant 7.0 \mathrm{mmol} / \mathrm{l}$ were regraded in a masked manner using an adaptation of the modified Airlie House Classification used in the Early Treatment Diabetic Retinopathy Study (ETDRS) ${ }^{14}$ Intra-grader reliability of our earlier grading has been reported. ${ }^{10}$ Questionable retinopathy lesions were adjudicated and other definite lesions reviewed by the senior authors (PM, LC), before the final retinopathy grade was determined. In grouping participants according to the retinopathy level, we followed the ETDRS summary, ${ }^{14}$ with an additional severity level (14: haemorrhages without microaneurysms; and 15: hard exudates, soft exudates, or intraretinal microvascular abnormalities, in the absence of any microaneurysms) to define presence of 'questionable' diabetic retinopathy. As in the WESDR, ${ }^{8}$ the retinopathy level for each participant was derived by concatenating the levels for the two eyes, giving the eye with the higher level greater weight, as shown in Table 1. This scheme provides a 15-step scale (10/10, 14-15/10, 14-15/14-15, 20/<20, 20/20, 35/ <35, $35 / 35,43 /<43,43 / 43,47 /<47,47 / 47,53 /<53,53 / 53$, $60+/<60+, 60+/ 60+)$, with all levels of proliferative retinopathy grouped as one step. The higher level of retinopathy for the two eyes thus determines the level of retinopathy for each participant.

For the purposes of this study, an increase of one or more steps on the 15-step scale over the 5-year period from the baseline examination was considered to indicate progression of retinopathy. Progression rates of two or more steps were also calculated. Retinopathy level was considered ungradable if the photographs from both eyes could not be graded or were missing, or if the photographs of one eye could not be graded (or was missing), whereas the fellow eye had retinopathy level $<20$.

\section{Statistical analysis}

Statistical analysis system (SAS, version 6.12, SAS

Institute Inc., Cary, NC, USA) was used to perform all 
Table 1 Adaptation from the ETDRS classification of diabetic retinopathy ${ }^{14}$

\begin{tabular}{llrr}
\hline Retinopathy level & Severity & \multicolumn{2}{c}{15 -Step scale } \\
\cline { 2 - 3 } & & Lower & Higher \\
\hline 10 & No retinopathy & $10 / 10$ & $14-15 / 14-15$ \\
$14-15$ & Questionable diabetic retinopathy & $14-15 / 10$ & $20 / 20$ \\
$20^{\mathrm{a}}$ & Minimal NPDR & $20 /<20$ & $35 / 35$ \\
$35^{\mathrm{a}}$ & Mild NPDR & $35 /<35$ & $43 / 43$ \\
$43^{\mathrm{a}}$ & Moderate NPDR & $43 /<43$ & $47 / 47$ \\
$47^{\mathrm{a}}$ & Moderately severe NPDR & $47 /<47$ & $53 / 53$ \\
$53^{\mathrm{a}}$ & Severe NPDR & $53 /<53$ & $60+/ 60+$ \\
$60^{\mathrm{a}}$ & PDR & $60+/<60+$ & \\
\hline
\end{tabular}

Abbreviations: NPDR, non-proliferative diabetic retinopathy; PDR, proliferative diabetic retinopathy.

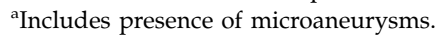

Table 2 Baseline characteristics of diabetic participants who (1) returned to 5-year examinations $(n=150)$, (2) did not return $(n=65)$, or (3) died $(n=69)$

\begin{tabular}{|c|c|c|c|c|c|}
\hline Baseline characteristics & $\begin{array}{c}\text { Participants } \\
(\mathrm{n}=150,147 \text { with } \\
\text { gradable photos) }\end{array}$ & $\begin{array}{l}\text { Non-participants } \\
(\mathrm{n}=65,61 \text { with } \\
\text { gradable photos) }\end{array}$ & *P-value & $\begin{array}{c}\text { Died } \\
(\mathrm{n}=69,60 \text { with } \\
\text { gradable photos) }\end{array}$ & *P-value \\
\hline Age (years) (mean $\pm S D)$ & $66.2 \pm 8.4$ & $65.9 \pm 8.3$ & NS & $73.6 \pm 7.3$ & 0.0001 \\
\hline Age at diagnosis (years) (mean \pm SD) & $60.4 \pm 11.2$ & $60.5 \pm 10.3$ & NS & $65.7 \pm 13.1$ & 0.005 \\
\hline Duration of diabetes (years) (mean \pm SD) & $5.8 \pm 7.5$ & $5.4 \pm 6.8$ & NS & $7.9 \pm 10.8$ & NS \\
\hline Fasting blood glucose $(\mathrm{mmol} / \mathrm{l})($ mean $\pm \mathrm{SD})$ & $8.1 \pm 3.3$ & $9.7 \pm 4.2$ & 0.009 & $9.5 \pm 4.5$ & 0.04 \\
\hline Systolic blood pressure $(\mathrm{mm} \mathrm{Hg})($ mean \pm SD) & $150.5 \pm 21.9$ & $150.6 \pm 20.9$ & NS & $155.0 \pm 29.7$ & NS \\
\hline Diastolic blood pressure $(\mathrm{mm} \mathrm{Hg})($ mean $\pm \mathrm{SD})$ & $83.4 \pm 10.9$ & $85.1 \pm 8.2$ & NS & $83.5 \pm 13.0$ & NS \\
\hline $\operatorname{Sex}(\mathrm{M}: \mathrm{F}) \%$ & 52.7: 47.3 & 44.6: 55.4 & NS & 60.9: 39.1 & NS \\
\hline Retinopathy present (number $(\%)$ ) & $50(34.0)$ & $23(37.7)$ & NS & $30(50.0)$ & 0.05 \\
\hline \multicolumn{6}{|l|}{ Retinopathy severity } \\
\hline None (number (\%)) & $97(64.7)$ & $38(58.5)$ & & $30(43.5)$ & \\
\hline NPDR (number (\%)) & $49(32.7)$ & $23(35.4)$ & & $27(39.1)$ & \\
\hline PDR (number (\%)) & $1(0.6)$ & $0(0)$ & & $3(4.4)$ & \\
\hline Ungradable (number $(\%)$ ) & $3(2.0)$ & $4(6.1)$ & & $9(13.0)$ & \\
\hline
\end{tabular}

Abbreviations: NPDR, non-proliferative diabetic retinopathy; NS, non-significant; PDR, proliferative diabetic retinopathy.

${ }^{*} P$-value in comparison with participants.

analyses, including $t$-tests, $\chi^{2}$-test, and logistic regression. Age, sex, fasting blood glucose level, and duration of diabetes were included in the multivariate logistic regression model. Odds ratios (OR) and 95\% confidence intervals (CI) are reported.

\section{Results}

At the baseline examination, a total of 284 participants had diabetes $(8.8 \%)$, including 218 who gave a history of diabetes and 66 whose diabetes was diagnosed from the fasting blood glucose test performed at the study. By the time the 5-year follow-up examination was conducted, $69(24.3 \%)$ had died, $40(14.1 \%)$ had moved from the study area, and $25(8.8 \%)$ refused to attend, 150 were re-examined, which represents $52.8 \%$ of all baseline participants with diabetes or $70 \%$ of 215 surviving participants with diabetes. Selected baseline characteristics of participants and non-participants (deaths and others) at the 5-year follow-up examination are shown in Table 2. Those who died were older, more likely to have retinopathy at baseline and had slightly higher fasting glucose levels than participants. Of others not examined, slightly higher fasting blood glucose was the only significant difference found between participants and non-participants $(P<0.01)$. Of the 284 baseline participants with diabetes, $24.3 \%$ died during the 5-year follow-up period, compared to a mortality rate of only $14.1 \%$ among persons without diabetes. Of subjects with diabetes who died, $46.9 \%$ had retinopathy at baseline, whereas $34.9 \%$ of survivors had retinopathy at baseline. The proportions with diabetic retinopathy were similar between baseline participants and those who returned to the 5-year examinations: that is, $103 / 284(36.3 \%)$ compared to $50 / 147(34.0 \%)$ returning participants with 
Table 3 Progression of retinopathy in 139 diabetic participants examined at both baseline and 5-year visits with retinal photographs available from both examinations

\begin{tabular}{lrcccc}
\hline Baseline retinopathy level & $n$ & DR absent (\%) & $\begin{array}{c}\text { NPDR questionable, } \\
\text { minimal, or mild (\%) }\end{array}$ & $\begin{array}{c}\text { NPDR moderate } \\
\text { or severe (\%) }\end{array}$ & $\begin{array}{c}\text { PDR (\%) } \\
\text { Macular } \\
\text { oedema (\%) }\end{array}$ \\
\hline DR absent & 90 & 77.8 & 22.2 & - & - \\
NPDR - quest, min, mild & 39 & 30.8 & 56.4 & 12.8 & 22.2 \\
NPDR - moderate, severe & 9 & - & 22.2 & 55.6 & 100 \\
PDR & 1 & 139 & & & 4.0 \\
Total & 5.3 & & & & \\
Macular oedema (\%) & & & & \\
\hline
\end{tabular}

Abbreviations: DR, diabetic retinopathy; $n$, number of participants with gradable photos; NPDR, non-proliferative diabetic retinopathy; PDR, proliferative diabetic retinopathy; quest, questionable diabetic retinopathy.

Table 4 Risk factors for progression of retinopathy level ${ }^{\mathrm{a}}$ in participants after 5-year follow-up

\begin{tabular}{lccc}
\hline Variable & $\begin{array}{c}\text { Progression } \\
\text { (mean } \pm \text { SD) }(\mathrm{n}=36)\end{array}$ & $\begin{array}{c}\text { No progression } \\
\text { (mean } \pm \text { SD) }(\mathrm{n}=103)\end{array}$ & $\begin{array}{c}\text { P-value } \\
\mathrm{b}\end{array}$ \\
\hline Age (years) & $66.7 \pm 8.2$ & $65.5 \pm 8.1$ & 0.45 \\
Duration of diabetes (years) & $7.4 \pm 6.4$ & $5.6 \pm 8.0$ & 0.17 \\
Systolic blood pressure (mm Hg) & $150 \pm 21$ & $150 \pm 23$ & 0.92 \\
Diastolic blood pressure (mm Hg) & $84 \pm 12$ & $83 \pm 11$ & 0.81 \\
Fasting blood glucose (mmol/l) & $9.5 \pm 4.3$ & $7.6 \pm 2.8$ & 0.02 \\
HDL Cholesterol (mmo/l) & $1.2 \pm 0.4$ & $1.3 \pm 0.4$ & 0.58 \\
Cholesterol (mmol/l) & $5.8 \pm 1.2$ & $5.7 \pm 1.0$ & 0.87 \\
Triglyceride (g/l) & $2.4 \pm 1.7$ & $2.3 \pm 1.3$ & 0.59 \\
Fibrinogen (g/l) & $3.8 \pm 0.9$ & $4.0 \pm 1.0$ & 0.24 \\
Body mass index (kg/m $\left.{ }^{2}\right)$ & $28.8 \pm 5.8$ & $27.9 \pm 4.8$ & 0.40 \\
Sex (\% female) & 44.4 & 48.5 & NS \\
Current smoking (\%) & 11.1 & 13.0 & NS \\
Hypertension definite (\%) & 44.4 & 54.4 & NS \\
Family history 1st generation $(\%)$ & 27.8 & 36.9 & NS \\
\hline
\end{tabular}

Abbreviations: HDL, high density lipoprotein; NS, non-significant.

aProgression of retinopathy level using the 15-step-scale in Table 1.

${ }^{b} t$-Tests for continuous variable, $\chi^{2}$ for categorical variable.

gradable retinal photographs had diabetic retinopathy at baseline.

Retinopathy developed in 20 of 90 participants with no diabetic retinopathy present at baseline $(22.2 \%, 95 \% \mathrm{CI}$ 14.1-32.2\%). After excluding those with other isolated retinopathy lesions but no microaneurysms, the 5-year cumulative incidence was only $14.4 \%$.

A total of 139 participants who had retinal photographs available at both the baseline and the 5-year examinations were considered for the analysis of progression of retinopathy, including progression from no retinopathy (step 10/10) to minimal or worse levels. Progression of diabetic retinopathy level in the worse eye of these 139 participants is shown in Table 3. The second column of this table presents the number of subjects with various retinopathy levels at baseline, and the remaining five columns represent the proportion with each retinopathy level 5 years later. Owing to the collapse of some retinopathy levels in Table 3, numbers with progression or regression calculated from this table are slightly different from the number and proportions described below, calculated according to retinopathy levels presented in Table 1. After the 5-year follow-up period, the retinopathy in $36(25.9 \%, 95 \%$ CI 18.8-34.0) had progressed one or more steps higher on the scale, 16 had regressed $(11.5 \%, 95 \%$ CI 6.7-18.0), and 87 remained at the same level as at the baseline examination $(62.6 \%$, 95\% CI 54.0-70.7). Among those participants who progressed after 5 years, $58.3 \%$ progressed by two or more steps (not shown in Table 3, owing to collapse of some levels in this table). Two of the nine participants $(22.2 \%)$ with moderate or severe non-proliferative retinopathy progressed to proliferative retinopathy. This number also accounted for $4.2 \%$ of participants with presence of any retinopathy lesion progressing to proliferative retinopathy.

Table 4 shows baseline characteristics of participants with and without progression of retinopathy level at 
Table 5 OR with confidence intervals (CI) of selected risk factors predicting progression of retinopathy in multivariate model

\begin{tabular}{|c|c|c|c|c|}
\hline \multirow[t]{2}{*}{ Risk factors } & \multicolumn{2}{|c|}{ Progression (\% (number)) } & \multirow{2}{*}{$\begin{array}{c}\text { OR }(95 \% \text { CI }) \\
\text { age-sex adjusted }\end{array}$} & \multirow{2}{*}{$\begin{array}{c}\text { OR }(95 \% \mathrm{CI}) \\
\text { multivariate model }\end{array}$} \\
\hline & With factor & Without factor & & \\
\hline Diabetes duration $>10$ years & $41.2(14)$ & $21.0(22)$ & $2.7(1.1-6.2)$ & $2.4(1.0-6.1)$ \\
\hline Fasting glucose $\geqslant 10.0$ & $44.4(12)$ & $21.3(23)$ & $3.3(1.3-8.4)$ & $3.2(1.3-8.4)$ \\
\hline
\end{tabular}

follow-up. The mean fasting glucose level was higher in those whose retinopathy progressed than in those without progression, $9.4 \pm 4.2$ vs $7.6 \pm 2.8 \mathrm{mmol} / \mathrm{l}$, respectively, $P<0.05$. Although diabetes duration, body mass index, and the proportion of women were all slightly higher among participants exhibiting progression, these differences were not statistically significant.

After adjusting for age and gender, fasting blood glucose level (OR 1.2, 95\% CI 1.1-1.4) and the duration of diabetes (OR 1.04, 95\% CI 1.0-1.1, per year) were the only statistically significant risk factors for progression. The relative risk for progression was even greater for participants with duration of diabetes $\geqslant 10$ years at follow-up (OR 2.7, 95\% CI 1.1-6.2) or for baseline fasting blood glucose levels $\geqslant 10 \mathrm{mmol} / 1$ (OR 3.4, 95\% CI 1.3-8.4). Other potential risk factors that were not statistically significantly related to progression were history of hypertension, serum cholesterol, triglycerides and HDL cholesterol, body mass index, and a first-degree family history of diabetes. The risk of progression was found to be 2.8 times higher (after 5 years of follow-up) when both fasting blood glucose $\geqslant 10 \mathrm{mmol} / 1$ and duration of diabetes $\geqslant 10$ years were present at the baseline examination (Table 5).

\section{Discussion}

There have been few similar community-based incidence studies of defined older population samples with which to compare these findings. Our cumulative incidence rate of $22.2 \%$ is somewhat lower than the cumulative incidence of $32.7 \%$ reported for non-insulin-treated diabetic participants aged $60-74$ years who were examined after 4 years in the WESDR. ${ }^{1}$ However, the rate from our study is identical to the 5-year incidence of $22.2 \%$ reported from a Japanese population sample aged 60 years or older ${ }^{6}$ and is very similar to the 4-year incidence rate of $19.2 \%$ reported from a Taiwanese study ${ }^{3}$ and to the 5-year incidence rate from a Korean study of older-onset diabetes. ${ }^{15}$ Our cumulative incidence rate is also close to $26.2 \%$ reported in a 4-year follow-up of a sample of non-Hispanic whites with a mean age of 59 years, ${ }^{16}$ and lower than the 4 -year rate of $30.8 \%$ incidence reported from a Swedish clinic study of 369 older-onset non-insulin-treated diabetic subjects. ${ }^{17}$ In a large Australian clinic-based study of 1210 persons with diabetes (all age groups), which achieved a $70 \%$ follow-up rate after 4 years, ${ }^{5}$ the incidence of new diabetic retinopathy lesions in those without retinopathy was $8 \%$ per person-year of follow-up, ${ }^{5}$ a somewhat higher projected rate than in the current study. This earlier study was reported from a period when the control of type II diabetes was typically poorer than in the last decade.

Although our diabetic sample is relatively small compared with many of these earlier studies, including the WESDR, ${ }^{1,8}$ its population-based design provides an unbiased estimate of the 5-year cumulative incidence of diabetic retinopathy in a community of persons aged 50 years or older.

Excluding retinopathy levels in which microaneurysms were absent was associated with a one-third decrease in the cumulative incidence to $14.4 \%$. Thus, the outcome of incidence studies may vary substantially depending on the classification of retinopathy used. Given that retinopathy with no microaneurysms present (termed 'questionable' diabetic retinopathy) could indicate the presence of resolving non-proliferative diabetic retinopathy, we grouped this questionable level with minimal and mild non-proliferative diabetic retinopathy levels as a separate entity.

Our 25.9\% 5-year cumulative rate of progression is relatively similar to 4-year follow-up rates found for non-Hispanic Whites (27.2\%) and Hispanics (23.0\%) aged $20-74$ years in the San Luis Valley Diabetic Study, ${ }^{16}$ and to the progression rate of $23.6 \%$ reported from the Japanese study ${ }^{6}$ and slightly lower than the $30.3 \%$ progression rate recorded in Taiwan. ${ }^{3}$ However, in comparison to the WESDR, our $15.1 \%$ rate for progression of two or more steps after 5 years is substantially lower than the $29.1 \%$ rate reported for progression in WESDR diabetic subjects aged 34-74 years after 4 years. $^{8}$ Although our study findings could be influenced by its relatively small number of diabetic subjects, it is also possible that this lower rate of progression could reflect improved diabetes management after the introduction of more widespread home glucose monitoring and awareness of the need to 
achieve tight diabetic control than was recommended a decade ago, before the publication of findings from the Diabetes Control and Complications Trial. ${ }^{18}$

We found that longer duration of diabetes and higher fasting blood glucose level were associated with increased progression of retinopathy over the 5-year period. This finding is not unexpected, because higher levels of fasting blood glucose were shown to be statistically significantly related to presence of moderate to severe retinopathy than to mild or no retinopathy in our previous prevalence report, after adjusting for age, gender, and the duration of diabetes. ${ }^{10}$

Our findings indicate that after 5 years, for individuals with diabetes for a period of 10 years or longer, the relative risk for progression of the retinopathy level by at least one step was 2.4 compared with the progression rate among individuals with a diabetes duration of less than 10 years. Similarly, for diabetic subjects with baseline fasting blood glucose levels $\geqslant 10 \mathrm{mmol} / \mathrm{l}$, the relative risk for progression of the retinopathy level by at least one step was 3.2 compared with the progression rate in diabetic subjects who were recorded as having fasting blood glucose levels less than $10 \mathrm{mmol} / 1$. These findings are consistent with recent data on glycaemic control in older type II diabetes subjects from the United Kingdom Prospective Diabetes Study. ${ }^{19}$

Strengths of this study include its population-based sample and stereo retinal photographic documentation of the retinopathy level, graded initially by LC and adjudicated by a retinal specialist (PM). The limitation of our study is a relatively low follow-up rate among participants with diabetes compared to an overall follow-up rate of $75 \%$ in the whole study sample. Of the 284 baseline participants with diabetes, 69 died and only 150 of $215(70.0 \%)$ survivors with diabetes returned at 5 years. Although this follow-up rate is acceptable, it could have introduced selection bias in estimating diabetic retinopathy incidence rates in the direction of either an overestimate or underestimate.

In summary, the cumulative incidence, after 5-year follow-up, of developing diabetic retinopathy lesions in this older community was $22.2 \%$. The 5 -year cumulative rate for retinopathy progression of at least one step or more was $25.9 \%$, whereas progression from any signs of non-proliferative retinopathy to proliferative retinopathy over this period was only $4.2 \%$. Higher baseline fasting blood glucose levels and longer duration of diabetes were the only statistically significant risk factors found for retinopathy progression after 5 years in this study.

\section{Acknowledgements}

This study was supported by the Australian National Health \& Medical Research Council (Grant no. 974159) and the Westmead Millennium Institute, University of Sydney.

\section{References}

1 Klein R, Klein BE, Moss SE, Davis MD, DeMets DL. The wisconsin epidemiologic study of diabetic retinopathy. $X$. Four-year incidence and progression of diabetic retinopathy when age at diagnosis is 30 years or more. Arch Ophthalmol 1989; 107: 244-249.

2 Klein R, Klein BE, Moss SE, Linton KL. The Beaver Dam Eye Study. Retinopathy in adults with newly discovered and previously diagnosed diabetes mellitus. Ophthalmology 1992; 99: 58-62.

3 Chen MS, Kao CS, Fu CC, Chen CJ, Tai TY. Incidence and progression of diabetic retinopathy among noninsulindependent diabetic subjects: a 4-year follow-up. Int $J$ Epidemiol 1995; 24: 787-795.

4 Dwyer MS, Melton III LJ, Ballard DJ, Palumbo PJ, Trautmann JC, Chu CP. Incidence of diabetic retinopathy and blindness: a population-based study in Rochester, Minnesota. Diabetes Care 1985; 8: 316-322.

5 Mitchell P. Development and progression of diabetic eye disease in Newcastle 1977-1984: rates and risk factors. Aust NZ J Ophthalmol 1985; 13: 39-44.

6 Morisaki N, Watanabe S, Kobayashi J, Kanzaki T, Takahashi $\mathrm{K}$, Yokote $\mathrm{K}$ et al. Diabetic control and progression of retinopathy in elderly patients: five-year follow-up study. J Am Geriatr Soc 1994; 42: 142-145.

7 Constable IJ, Welborn TA, Cooper RL, McCann VJ, Stanton KM, Greer DV et al. Medical correlates and diabetic retinopathy screening. Trans Ophthalmol Soc UK 1980; 100: 78-82.

8 Klein R, Klein BE, Moss SE, Cruickshanks KJ. The Wisconsin Epidemiologic Study of diabetic retinopathy. XIV. Ten-year incidence and progression of diabetic retinopathy. Arch Ophthalmol 1994; 112: 1217-1228.

9 Attebo K, Mitchell P, Smith W. Visual acuity and the causes of visual loss in Australia. The Blue Mountains Eye Study. Ophthalmology 1996; 103: 357-364.

10 Mitchell P, Smith W, Wang JJ, Attebo K. Prevalence of diabetic retinopathy in an older community. The blue mountains eye study. Ophthalmology 1998; 105: 406-411.

11 Mitchell P, Wang JJ, Foran S, Smith W. Five-year incidence of age-related maculopathy lesions: the blue mountains eye study. Ophthalmology 2002; 109: 1092-1097.

12 Foran S, Wang JJ, Mitchell P. Causes of incident visual impairment: the blue mountains eye study. Arch Ophthalmol 2002; 120: 613-619.

13 The Expert Committee on the Diagnosis and Classification of Diabetes Mellitus. Report of the expert committee on the diagnosis and classification of diabetes mellitus. Diabetes Care 1998; 21: S5-S19.

14 Davis MD, Fisher MR, Gangnon RE, Barton F, Aiello LM, Chew EY et al. Risk factors for high-risk proliferative diabetic retinopathy and severe visual loss: Early Treatment Diabetic Retinopathy Study Report 18. Invest Ophthalmol Vis Sci 1998; 39: 233-252.

15 Kim HK, Kim CH, Kim SW, Park JY, Hong SK, Yoon YH et al. Development and progression of diabetic retinopathy in Koreans with NIDDM. Diabetes Care 1998; 21: 134-138. 
16 Tudor SM, Hamman RF, Baron A, Johnson DW, Shetterly $\mathrm{SM}$. Incidence and progression of diabetic retinopathy in Hispanics and non-Hispanic whites with type 2 diabetes. San Luis Valley Diabetes Study, Colorado. Diabetes Care 1998; 21: 53-61.

17 Agardh E, Agardh CD, Koul S, Torffvit O. A four-year follow-up study on the incidence of diabetic retinopathy in older onset diabetes mellitus. Diabetic Med 1994; 11: 273-278.
18 Progression of retinopathy with intensive versus conventional treatment in the Diabetes Control and Complications Trial. Diabetes Control and Complications Trial Research Group. Ophthalmology 1995; 102: 647-661.

19 Stratton IM, Kohner EM, Aldington SJ, Turner RC, Holman RR, Manley SE et al. UKPDS 50: risk factors for incidence and progression of retinopathy in Type II diabetes over 6 years from diagnosis. Diabetologia 2001; 44: 156-163. 